\title{
Analysis of bivariate coupling by means of recurrence
}

\author{
Christoph Bandt ${ }^{1}$, Andreas Groth ${ }^{1}$, Norbert Marwan ${ }^{2}$, M. Carmen \\ Romano $^{2,3}$, Marco Thiel ${ }^{2,3}$, Michael Rosenblum ${ }^{2}$, and Jürgen Kurths ${ }^{2}$ \\ 1 Institute of Mathematics, University of Greifswald; Germany \\ bandt@uni-greifswald.de \\ 2 Institute of Physics, University of Potsdam, Germany \\ 3 Department of Physics, University of Aberdeen, UK
}

Summary. We describe dependencies in bivariate time series by appearance of equal or similar recurrence patterns. Various recurrence concepts and parameters are discussed. They are applied to detect synchronization in model systems of coupled oscillators and coupling in EEG data.

Key words: time series analysis, nonlinear dynamics, recurrence, synchronization

\section{Introduction}

In the analysis of coupled systems, various techniques have been developed to model and detect dependencies from observed bivariate time series. Most well-founded methods, like Granger causality and partial coherence, are based on the theory of linear systems: on correlation functions, spectra and vector autoregressive processes. In this paper we discuss a non-linear approach using recurrence.

Recurrence, which intuitively means the repeated occurrence of a very similar situation, is a basic notion in dynamical systems. The classical theorem of Poincaré says that for every dynamical system with an invariant probability measure $P$, almost every point in a set $B$ will eventually return to $B$. Moreover, for ergodic systems the mean recurrence time is $1 / P(B)$ [1]. Details of recurrence patterns were studied when chaotic systems came into the focus of research, and it turned out that they are linked to Lyapunov exponents, generalized entropies, the correlation sum, and generalized dimensions $[2,3]$.

Our goal here is to develop methods for time series which typically contain a few hundreds or thousands of values and which need not come from a stationary source. While Poincaré's theorem holds for stationary stochastic processes, and linear methods require stationarity at least for sufficiently large windows, recurrence methods need less stationarity. We outline different con- 
cepts of recurrence by specifying different classes of sets $B$. Then we visualize recurrence and define recurrence parameters similar to autocorrelation.

We are going to apply recurrence to the analysis of bivariate data. The basic idea is that coupled systems show similar recurrence patterns. We can study joint recurrences as well as cross recurrence. We shall see that both approaches have their benefits and drawbacks.

Model systems of coupled oscillators form a test bed for analysis of bivariate time series since the corresponding differential equations involve a parameter which precisely defines the degree of coupling. Changing the parameter we can switch to phase synchronization and generalized synchronization. The approaches of cross and joint recurrence are compared for several models. In view of possible experimental requirements, recurrence is studied on ordinal scale as well as on metric scale. Several quantities for the description of synchronization are derived and illustrated. Finally, two different applications to EEG data will be presented.

\section{Recurrence on different scales}

\section{Nominal scale}

We start with an ordinary time series of numbers $x_{1}, x_{2}, \ldots, x_{N}$. Recurrence basically means that certain numbers will repeat: $x_{i}=x_{j}$. This is the proper concept when the values $x_{i}$ form a nominal scale - they are just symbols from a finite or countable alphabet. A typical example is the nucleotide sequence of a DNA segment, with values A,C,G and T (which we can code 1,2,3,4). Since letters will repeat very often, we usually prescribe a length $d$ for the word which should repeat:

$$
x_{i+n}=x_{j+n}, \quad n=0, \ldots, d-1 .
$$

Here $d$ is a parameter which indicates the strength of recurrence. Finding occurrences of words in large data is a basic algorithmic task in bioinformatics. The statistical structure of such sequences is modelled by Hidden Markov Models, also called probabilistic automata [4].

\section{Metric scale}

If the $x_{i}$ are real numbers, instead of $x_{i}=x_{j}$ we require that $x_{j}$ is in the vicinity or neighborhood of $x_{i}$ :

$$
\left|x_{i}-x_{j}\right| \leq \varepsilon,
$$

where $\varepsilon$ is a predefined threshold. According to the ergodic theorem mentioned above, the mean recurrence time is of order $1 / \varepsilon$ which gives a clue on how to choose $\varepsilon$. 
Due to different density of the values, different $x_{i}$ will have different numbers of neighbors. This can be mended by taking rank numbers

$$
r_{i}=\#\left\{k \mid 1 \leq k \leq N, x_{k}<x_{i}\right\}
$$

instead of the $x_{i}$, and integer $\varepsilon$. Then each $x_{i}$ (except for the $\varepsilon$ largest and smallest values) has $2 \varepsilon$ recurrences. Eckmann et al [5] used constant number of neighbors when they introduced recurrence plots.

However, it makes little sense to require that only single values repeat. For the function $\sin t, t \geq 0$ the value 0 repeats at $t=\pi$, but this is a false neighbor, proper recurrence (in fact periodicity) appears at $2 \pi$. Thus we shall again choose a strength parameter $d$ and require

$$
\left|x_{i+n}-x_{j+n}\right| \leq \varepsilon, \quad n=0, \ldots, d-1 .
$$

\section{Vector recurrence}

The last condition can also be interpreted in a different way. We take the $d$-dimensional vectors $\boldsymbol{x}_{i}=\left(x_{i}, x_{i+1}, \ldots, x_{i+d-1}\right) \in \mathbb{R}^{d}$ and consider their approximate equality

$$
\left\|\boldsymbol{x}_{i}-\boldsymbol{x}_{j}\right\| \leq \varepsilon,
$$

with respect to the maximum norm in $\mathbb{R}^{d}$. However, it is also possible to consider any other norm on $\mathbb{R}^{d}$, like the Euclidean norm, or similarity indices like cosine similarity and the Mahalanobis distance. The choice of the distance function and the threshold (e.g. fixed, time-dependent, fixed amount of nearest neighbors) depends on the particular problem under consideration. For an overview we refer to [3].

Vector recurrence is certainly the appropriate concept when our time series does not consist of numbers but of vectors. This is the case for multivariate time series treated below, in particular for $d$-dimensional time series obtained numerically from a model system of $d$ differential equations. For such systems, studied in Sections 5 and 6, we need a slightly different notation.

\section{Differential equations and delay embedding}

In the formalism of differentiable dynamical systems, the state of a system at time $t$ is described by a vector

$$
\boldsymbol{x}(t)=\left[x_{1}(t), x_{2}(t), \ldots, x_{d}(t)\right] \in \mathbb{R}^{d},
$$

where $x_{n}(t)$ denotes the $n$-th component at time $t$. The evolution of the state of the system in time, i.e., its trajectory, is determined by a flow $F(\cdot)$, such that $\dot{\boldsymbol{x}}(t)=F(\boldsymbol{x}(t))$. The components $x_{n}(t)$ of the state vector $\boldsymbol{x}(t)$ are observable physical variables, such as the position and velocity of a particle. However, in an experimental setting typically not all relevant components are known or 
can be measured. If certain conditions are fulfilled, it is possible to reconstruct the trajectory of the system from a scalar measurement $u(t)=f[\boldsymbol{x}(t)]$, e.g., by means of its delay embedding $[6,7]$

$$
\boldsymbol{u}(t)=[u(t), u(t+\vartheta), \ldots, u(t+(m-1) \vartheta)] \in \mathbb{R}^{m}
$$

where $\vartheta$ denotes the time delay and $m$ the embedding dimension. In the ideal case there is a functional relationship (strictly speaking, a diffeomorphism) between the original unknown components and those of the delay embedding.

Although the underlying system evolves continuously in time, we measure the system at discrete time points $i \Delta t$, where $i=1, \ldots, N$ and $\Delta t$ is the sampling rate. When confusion is possible, we denote by $\boldsymbol{x}_{i}=\boldsymbol{x}(i \Delta t)$ and $\boldsymbol{u}_{i}=$ $\boldsymbol{u}(i \Delta t)$ the points of the original and reconstructed trajectory, respectively. Otherwise we use $\boldsymbol{x}_{i}$ for both, as in (1).

\section{Remarks on dynamical systems}

Periodicity is an extreme case of recurrence. And in deterministic systems, an exact recurrence to a state $\boldsymbol{x}_{i}$ at a later time point $j$ is only possible in the case of periodic dynamics. Otherwise, the required uniqueness of the solution of a dynamical system is not fulfilled.

In our definition (1) of vector recurrence, the recurrence set $B$ is a ball of radius $\varepsilon$ around $\boldsymbol{x}_{i}$ with respect to the given norm on $\mathbb{R}^{d}$. In case of the maximum norm it is a cube of side length $2 \varepsilon$. These sets are not disjoint for different $i$. It may happen that $j$ realizes a recurrence to both $i_{1}$ and $i_{2}$ but $i_{2}$ does not represent a recurrence to $i_{1}$. Thus sometimes one might wish the recurrence sets to form a disjoint partition of phase space, $\mathbb{R}^{d}=B_{1} \cup \ldots \cup B_{k}$.

When we decompose the phase space into regions and assign the same symbol to all states within one region, our metric vector data become nominal data (symbolization), and we neglect all further information about distances. This coarse-graining leads to a lower level of resolution, but on the other hand also to a weaker stationarity condition on the measurement. Note that for recurrence on a metric scale, stationarity is only required up to a threshold $\varepsilon$. By varying this threshold, and in case of $\boldsymbol{u}_{i}$ also the embedding dimension, we are able to balance between resolution and stationarity requirements.

In the sequel, we give a partial answer to the question for an appropriate decomposition of phase space.

\section{Ordinal scale}

The ordinal scale of numbers is between the metric and the nominal one: the order structure of the states is known, but no meaningful distance of the values is defined. The analysis on an ordinal scale, in contrast to the one on a metric scale, is invariant with respect to a strictly monotonic transformation. The classification of levels of measurement resolution into metric, ordinal 
and nominal, was originally proposed in statistics [8]. Here, we suggest this approach for the analysis of dynamical systems.

We consider two states $\boldsymbol{u}_{i}$ and $\boldsymbol{u}_{j}$ in the reconstructed phase space. Order patterns are related to the time-delayed embedding (3). They will not be applied to the systems (2). We define recurrence on the ordinal scale if both states exhibit the same order structure

$$
\pi\left(\boldsymbol{u}_{i}\right)=\pi\left(\boldsymbol{u}_{j}\right),
$$

where $\pi$ is a mapping function that encodes the order structure.

To illustrate this idea, suppose that the reconstructed trajectory has embedding dimension $m=2$ and time delay $\vartheta$. In this case, two relationships between $u_{i}$ and $u_{i+\vartheta}$ are possible, apart from equality. ${ }^{4}$ We encode the order structure as a new symbol

$$
\pi\left(\boldsymbol{u}_{i}\right)= \begin{cases}0: & u_{i}<u_{i+\vartheta} \\ 1: & u_{i}>u_{i+\vartheta}\end{cases}
$$

where $\pi$ is called order pattern of $\boldsymbol{u}_{i}$. Thus the phase space is divided by the identity into two areas (Fig. 1). This way of generating a new symbol sequence

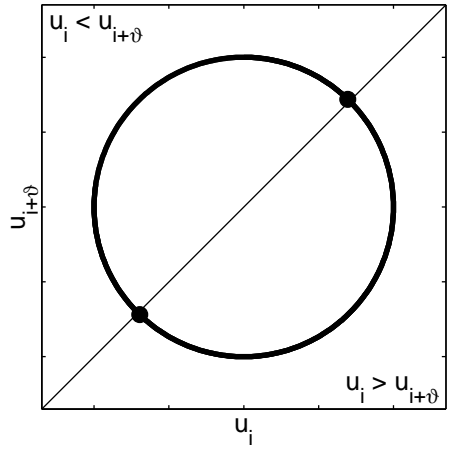

Fig. 1. Periodic trajectory in phase space and decomposition of the phase space $\left(u_{i}, u_{i+\vartheta}\right)$ by order patterns.

is common in statistics (e.g. $[9,10,11])$. Our approach was originally motivated by Kendall's tau-correlation [12], which was modified to an auto-correlation function for time series $[13,14]$. In classical time series analysis there are practically no methods which use order patterns of higher dimensions. Here we can use order patterns of length $d$, so that we have again a parameter for the strength of recurrence, as well as more complicated order patterns [15].

\footnotetext{
${ }^{4}$ In general we neglect the equality of values. This is reasonable if we consider systems with continuous distribution of the values, where equality has measure zero.
} 


\section{Order patterns of length 3}

Let us consider embedding dimension $m=3$, which is related to the phase of an oscillator [16], discussed in Section 5. Here the phase space is nicely decomposed into $m !=6$ regions. These regions are separated by planes of pairwise equalities $\left(u_{i}=u_{i+\vartheta}, u_{i+\vartheta}=u_{i+2 \vartheta}, u_{i}=u_{i+2 \vartheta}\right)$ and are arranged around the main diagonal $u_{i}=u_{i+\vartheta}=u_{i+2 \vartheta}$ (Fig. 2). All states $\boldsymbol{u}_{i}$ within
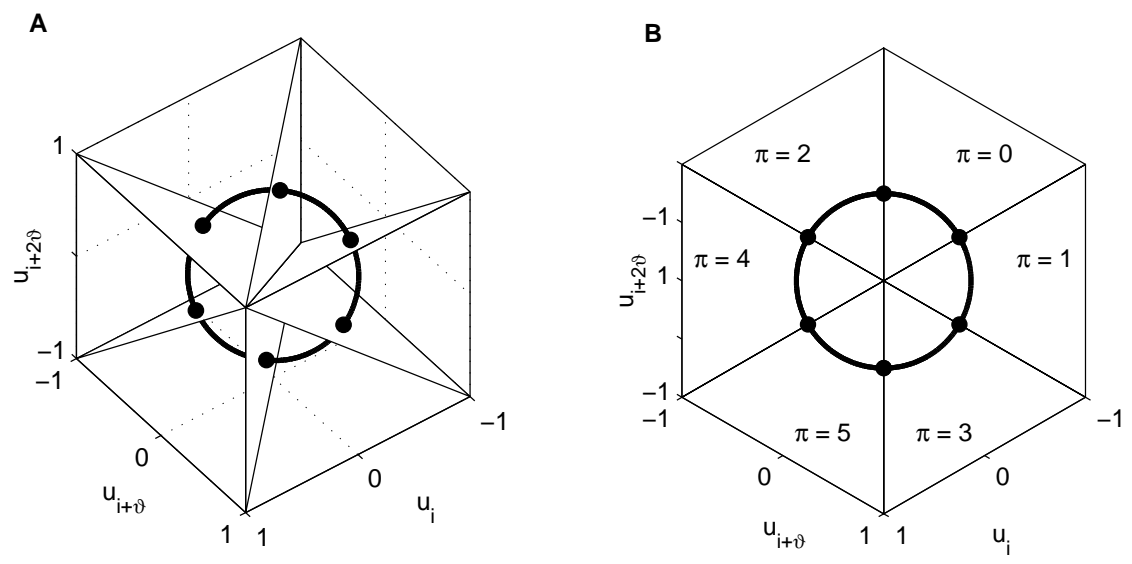

Fig. 2. (a) Decomposition of the phase space $\left(u_{i}, u_{i+\vartheta}, u_{i t+2 \vartheta}\right)$ by order patterns $\pi$ and possible trajectory of a sine function. (b) Same plot with viewing angle in direction of the main diagonal

a single region of the phase space have the same structure of order relations. Hence, they are associated to the same symbol $\pi\left(\boldsymbol{u}_{i}\right)$ (Fig. 3).

$u_{i}$

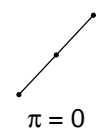

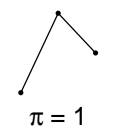
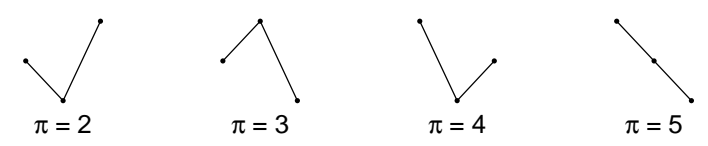

Fig. 3. The vector $\boldsymbol{u}_{i}=\left(u_{i}, u_{i+\vartheta}, u_{i t+2 \vartheta}\right)$ in reconstructed phase space can form six different order patterns. The labelling is not important for the analysis and is added just for illustration.

This scheme of mapping states $\boldsymbol{u}_{i}$ to symbols $\pi\left(\boldsymbol{u}_{i}\right)$ works for arbitrary dimension $m$. The phase space decomposition into $m$ ! regions is related to the concept of permutation entropy [17] which for various dynamical systems agrees with the metric entropy $[18,19]$. 

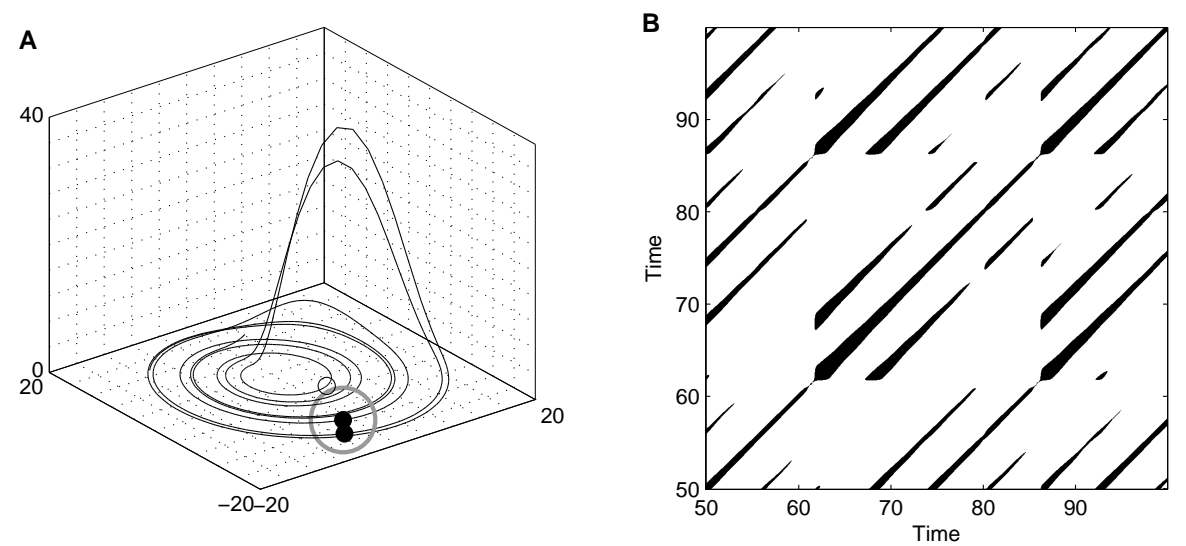

Fig. 4. (A) Segment of the phase space trajectory of the Rössler system, Eqs. (14), with $a=0.15, b=0.20, c=10$, using the components $(x, y, z)$. (B) The corresponding recurrence plot based on metric scale. A phase space vector at $j$ which falls into the neighborhood (grey circle in (A)) of a given phase space vector at $i$ is considered to be a recurrence point (black point on the trajectory in (A)). This is marked by a black point in the $\mathrm{RP}$ at the position $(i, j)$. A phase space vector outside the neighborhood (empty circle in (A)) leads to a white point in the RP. The radius of the neighborhood for the $\mathrm{RP}$ is $\varepsilon=5 ; L_{2}$-norm is used.

\section{Recurrence plots}

\section{Univariate recurrence}

Given a trajectory $\left\{\boldsymbol{x}_{i}\right\}_{i=1}^{N}$ of a dynamical system in phase space, we can compute its recurrence matrix, i.e., the time indices $j$ at which the trajectory recurs to the state $\boldsymbol{x}_{i}$, for $i, j=1, \ldots, N$. Hence, the recurrence matrix is a binary $N \times N$ matrix with entry $\mathbf{R}_{i, j}=1$ if the trajectory at time j recurs to the state $\boldsymbol{x}_{i}$ and entry $\mathbf{R}_{i, j}=0$, otherwise.

As mentioned above, recurrence can be defined on a metric, nominal or ordinal scale. Accordingly, the recurrence matrix on a metric scale is

$$
\mathbf{R}_{i, j}=\Theta\left(\varepsilon-\left\|\boldsymbol{x}_{i}-\boldsymbol{x}_{j}\right\|\right)
$$

on a nominal scale

$$
\mathbf{R}_{i, j}=\delta\left(\boldsymbol{x}_{i}-\boldsymbol{x}_{j}\right)
$$

and on an ordinal scale

$$
\mathbf{R}_{i, j}=\delta\left(\pi\left(\boldsymbol{u}_{i}\right)-\pi\left(\boldsymbol{u}_{j}\right)\right)
$$

where $\Theta(x)=1$ for $x \geq 0$ and $\Theta(x)=0$ else, $\delta(x)=1$ if $x=0$ and $\delta(x)=0$ otherwise, and $i, j=1, \ldots, N$. 

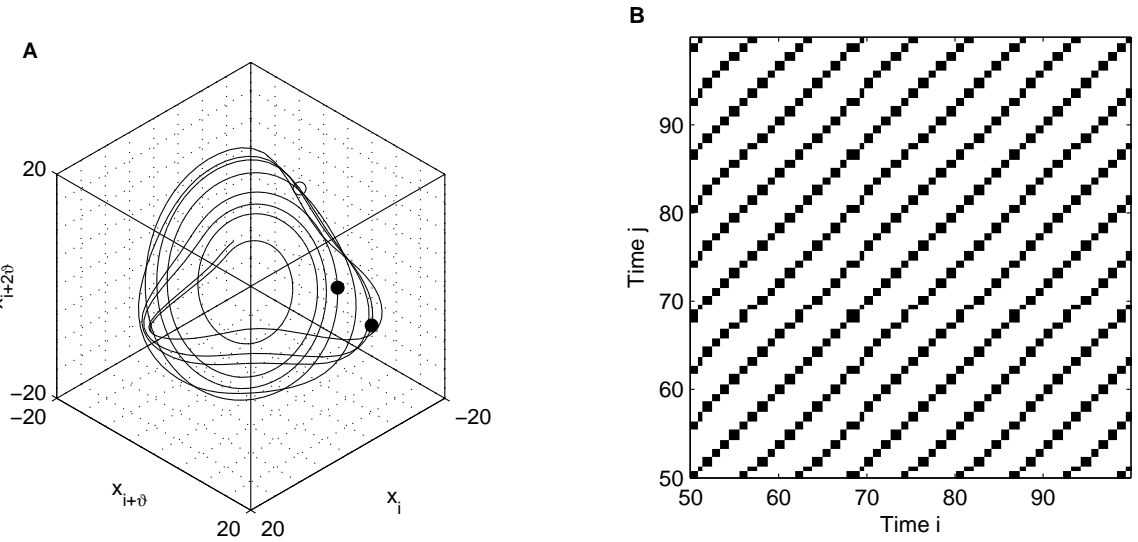

Fig. 5. (A) Segment of the phase space trajectory of the Rössler system, Eqs. (14), with $a=0.15, b=0.20, c=10$, by using its time-delayed first component $\left(x_{i}, x_{i+\vartheta}, x_{i+2 \vartheta}\right)$ with $\vartheta=2$ and (B) its corresponding recurrence plot based on ordinal scale. Phase space vectors in the same region are considered as recurrent points (black points on the trajectory in $(\mathrm{A})$ ), where a phase space vector in a different region (empty circle in $(\mathrm{A})$ ) is not a recurrence point.

A recurrence plot $(\mathrm{RP})$ is the graphical representation of a recurrence matrix [20,3]. The RP is obtained by plotting the recurrence matrix and using different colors for its binary entries, e.g., plotting a black dot at the coordinates $(i, j)$, where $\mathbf{R}_{i, j}=1$, and a white dot, where $\mathbf{R}_{i, j}=0$. Both axes of the RP are time axes. Since $\mathbf{R}_{i, i} \equiv 1$ for $i=1 \ldots N$ by definition, the $\mathrm{RP}$ has always a black main diagonal line. Furthermore, the RP is symmetric with respect to the main diagonal, i.e. $\mathbf{R}_{i, j}=\mathbf{R}_{j, i}$.

RPs yield important insights into the time evolution of phase space trajectories, because typical patterns in RPs are linked to a specific behavior of the system. One important structural element are diagonal lines $\mathbf{R}_{i+k, j+k}=1$ for $k=0 \ldots l-1$, where $l$ is the length of the diagonal line. On metric scale a diagonal occurs when a segment of the trajectory runs almost in parallel to another segment (i.e. through an $\varepsilon$-tube around the other segment) for $l$ time units (cf. Fig. 4). The length of this diagonal line is determined by the duration of such similar local evolution of the trajectory segments. The direction of these diagonal structures is parallel to the main diagonal. Since the definition of the Rényi entropy of second order $K_{2}$ is based on how long trajectories evolve within an $\varepsilon$-tube, it is possible to estimate $K_{2}$ by means of the distribution of diagonal lines in the RP $[21,3]$. On an ordinal scale we also obtain diagonal structures when two different segments of the trajectory have the same sequence of order patterns (cf. Fig. 5). In particular, we will show how these diagonal lines are linked to the phase of an oscillator and will derive a measure to quantify phase synchronization. 


\section{Bivariate recurrence plots}

There are two approaches to extend RPs to the analysis of bivariate data $\left(\boldsymbol{x}_{i}, \boldsymbol{y}_{i}\right)$. In the first approach, the common auto-reccurences are registered. This is essentially the same procedure as going from metric recurrence to vector recurrence in Section 2, and it can be done for two qualitatively different systems: the vectors $\boldsymbol{x}_{i}$ and $\boldsymbol{y}_{i}$ can have different dimension, and represent different physical quantities.

In the other approach, we compute the recurrence of the states of one system to the other, i. e. the distances between the different systems in phase space. This requires a certain degree of similarity of the systems for $\boldsymbol{x}$ and $\boldsymbol{y}$ although the ordinal approach also allows to compare physical different systems. However, lagged dependencies can be better visualized, and the two time series can have different length. As we will show here, depending on the situation, one approach might be more appropriate than the other.

\section{Joint recurrence plots}

The first possibility to compare $\boldsymbol{x}, \boldsymbol{y}$ is to consider the recurrences of their trajectories in their respective phase spaces separately and regard the times at which both of them recur simultaneously, i.e. when a joint recurrence occurs $[22,3]$. A joint recurrence plot (JRP) is defined as pointwise product of the two RPs of the two considered systems

$$
\mathbf{J R}_{i, j}^{\boldsymbol{x}, \boldsymbol{y}}=\mathbf{R}_{i, j}^{\boldsymbol{x}} \cdot \mathbf{R}_{i, j}^{\boldsymbol{y}}, \quad i, j=1, \ldots, N .
$$

In this approach, a recurrence takes place if the first system at time $j$ described by the vector $\boldsymbol{x}_{j}$ returns to the neighborhood of a former point $\boldsymbol{x}_{i}$, and simultaneously the second system $\boldsymbol{y}_{j}$ returns at the same time $j$ to the neighborhood of a formerly visited point $\boldsymbol{y}_{i}$.

Actually, joint recurrence is just the vector recurrence of the bivariate series $\left(\boldsymbol{x}_{i}, \boldsymbol{y}_{i}\right)_{i=1, \ldots, N}$. The dimensions of the vectors $\boldsymbol{x}$ and $\boldsymbol{y}$ can differ, and we can consider different norms and different thresholds $\varepsilon$ for each system, so that the recurrence conditions can be adapted to each system separately, respecting the corresponding natural measure. Mathematically, this just means taking the norm $\|(\boldsymbol{x}, \boldsymbol{y})\|=\max \left\{\|\boldsymbol{x}\|_{1} / \varepsilon_{1},\|\boldsymbol{y}\|_{2} / \varepsilon_{2}\right\}$ on the product space of the two phase spaces.

We mention that a product representation similar to (9) holds for the transition from ordinary recurrence to the recurrence of $m$ successive states: $\mathbf{R}_{i, j}^{x}=\prod_{k=0}^{m-1} \mathbf{R}_{i+k, j+k}^{x}$, which simplifies plots for recurrence of strength $m$.

A delayed version of the joint recurrence matrix can be introduced by

$$
\mathbf{J R}_{i, j}^{\boldsymbol{x}, \boldsymbol{y}}=\mathbf{R}_{i, j}^{\boldsymbol{x}} \cdot \mathbf{R}_{i+\tau, j+\tau}^{\boldsymbol{y}}, \quad i, j=1, \ldots, N-\tau,
$$

which is useful for the analysis of interacting delayed systems (e.g. for lag synchronization) $[23,24]$, and for systems with feedback. 


\section{Cross recurrence plots}

A cross recurrence plot (CRP) visualizes dependencies between two different systems by looking at recurrences from one system to the other [25, 3]. Using a metric scale, it is defined as

$$
\mathbf{C R}_{i, j}^{\boldsymbol{x}, \boldsymbol{y}}=\Theta\left(\varepsilon-\left\|\boldsymbol{x}_{i}-\boldsymbol{y}_{j}\right\|\right),
$$

for the nominal scale it is

$$
\mathbf{C R}_{i, j}^{\boldsymbol{x}, \boldsymbol{y}}=\delta\left(\boldsymbol{x}_{i}-\boldsymbol{y}_{j}\right),
$$

and for the ordinal scale

$$
\mathbf{C R}_{i, j}^{\boldsymbol{u}, \boldsymbol{v}}=\delta\left(\pi\left(\boldsymbol{u}_{i}\right)-\pi\left(\boldsymbol{v}_{j}\right)\right),
$$

with $i=1, \ldots, N, j=1, \ldots, M$. The length of the trajectories of $\boldsymbol{x}$ and $\boldsymbol{y}$, or $\boldsymbol{u}$ and $\boldsymbol{v}$, respectively, need not be identical, so that $\mathbf{C R}$ need not be a square matrix. However, in the metric case both systems must be represented in the same phase space, otherwise we cannot measure distances between states of both systems. Therefore, the data under consideration should be from very similar processes and, actually, should represent the same observable.

On ordinal or nominal scale, this is not necessary. Nevertheless, we have to take the same embedding dimension for the delay vectors to define order patterns of the same length, or meaningful related decompositions with equal number of sets when symbolization is used to obtain a nominal series.

Since the values of the main diagonal $\mathbf{C R}_{i, i}$ for $i=1 \ldots N$ are not necessarily one, there is usually no black main diagonal. The lines which are diagonally oriented are here of major interest, too. They represent segments on both trajectories, which run parallel for some time. The distribution and length of these lines are obviously related to the interaction between the dynamics of both systems. A measure based on the lengths of such lines can be used to find nonlinear interrelations between both systems (Section 4).

\section{Comparison between CRPs and JRPs}

In order to illustrate the difference between CRPs and JRPs, we consider the trajectory of the Rössler system [26]

$$
\begin{aligned}
& \dot{x}=-y-z, \\
& \dot{y}=x+a y, \\
& \dot{z}=b+z(x-c),
\end{aligned}
$$

in three different situations: the original trajectory (Fig. 6A), the trajectory rotated around the $z$-axis (Fig. $6 \mathrm{~B}$ ) and the trajectory under the time scale transformation $\tilde{t}=t^{2}$, which gives the same picture as the first one. 
Let us consider the RPs of these three trajectories. The RP of the original trajectory is identical to the RP of the rotated one, (Fig. 7A) but the RP of the stretched/compressed trajectory is different from the RP of the original trajectory (Fig. 7B): it contains bowed lines, as the recurrent structures are shifted and stretched in time with respect to the original RP.

A

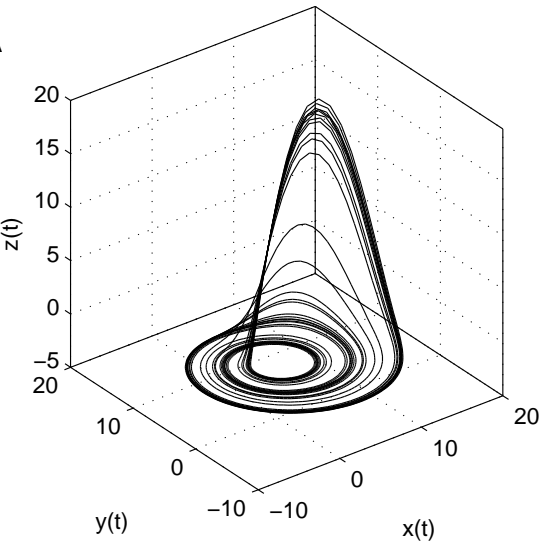

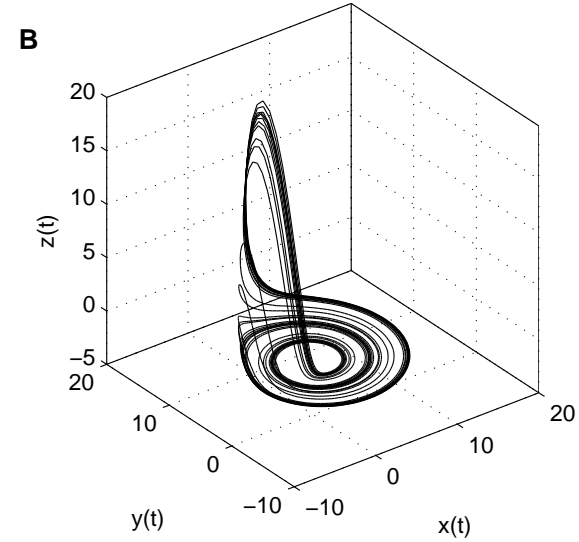

Fig. 6. Phase space trajectories of the Rössler system (Eqs. 14, with $a=0.15$, $b=0.2$ and $c=10)$ : (A) original system, (B) rotated around the $z$-axis by $\frac{3}{5} \pi$.

Now we calculate the CRP between the original trajectory and the rotated one (Fig. 7C) and observe that it is rather different from the RP of the original trajectory (Fig. 7A). This is because in CRPs the difference between each pair of vectors is computed, and this difference is not invariant under rotation of one of the systems. Hence, CRPs do not detect that both trajectories are identical up to a rotation. In contrast, the JRP of the original trajectory and the rotated one is identical to the RP of the original trajectory (Fig. 7A). This is because JRPs consider joint recurrences, and the recurrences of the original and the rotated system are identical.

The CRP between the original trajectory and the stretched/compressed one contains bowed lines, which reveals the functional shape of the parabolic transformation of the time scale (Fig. 7D) [3]. Note that CRPs represent the times at which both trajectories visit the same region of the phase space. On the other hand, the JRP of these trajectories - the intersection of the black sets in Fig. 7A and Fig. 7B - is almost empty, except for the main diagonal, because the recurrence structure of both systems is so different. There are almost no joint recurrences. Therefore, JRPs are not built to detect a time transformation applied to the trajectory, even though the shape of the phase space trajectories is identical.

To conclude, we can state that CRPs are more appropriate to investigate relationships between the parts of the same system which have been subjected 
A

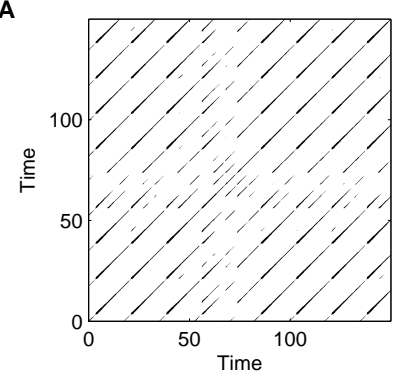

C

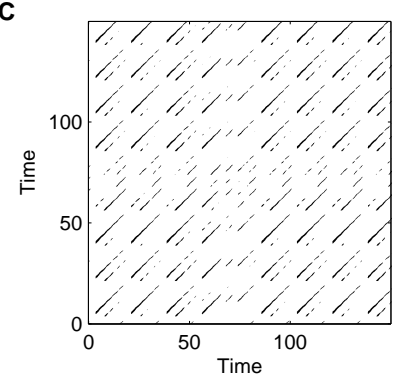

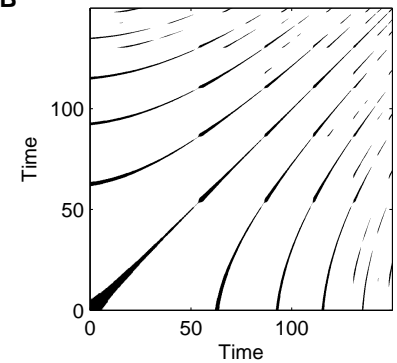

D

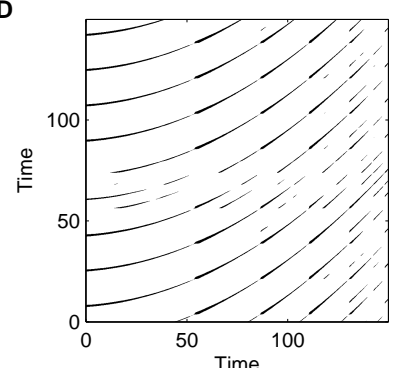

Fig. 7. RPs of (A) the original trajectory of the Rössler system and (B) the stretched/compressed trajectory. (C) CRP of the original and rotated trajectories and (D) CRP of the original and stretched/compressed trajectories. The threshold for recurrence is $\varepsilon=2$.

to different physical or mechanical processes, e.g., two borehole cores in a lake subjected to different compression rates. On the other hand, JRPs are more appropriate for the investigation of two interacting systems which influence each other, and hence, adapt to each other, e.g., in the framework of phase and generalized synchronization.

\section{Quantification of recurrence}

\section{Auto-recurrence}

The diagonals parallel to the main diagonal represent different epochs of phase space trajectories which evolve in a similar manner. Therefore, as a first approach we introduce some measures quantifying the density of recurrence points and the length of diagonal lines in dependence on their distance to the main diagonal [3].

The density of points on a certain diagonal with distance $\tau$ from the main diagonal is the auto-recurrence rate

$$
R R_{\tau}=\frac{1}{N-\tau} \sum_{i=1}^{N-\tau} \mathbf{R}_{i, i+\tau}
$$


where $\tau>0$ corresponds to diagonals above and $\tau<0$ to diagonals below the main diagonal, which represent positive and negative time delays, respectively. The auto-recurrence rate can be considered as a non-linear version of the autocorrelation function, since it also describes higher order correlations between the points of the trajectory in dependence on $\tau$ [27]. It can be interpreted as an estimate of the probability $p(\tau)$ that a state recurs to its $\varepsilon$-neighborhood after $\tau$ time steps.

Similar to auto-correlation, auto-recurrence fulfils $R R_{0}=1$ and is symmetric: $R R_{\tau}=R R_{-\tau}$. However, $R R_{\tau}$ is always between 0 and 1 . The reference line, which corresponds to the zero line for correlation functions, is given by the average recurrence rate

$$
R R=\frac{1}{N^{2}} \sum_{i, j=1}^{N} \mathbf{R}_{i, j}
$$

It is clear that $R R$ and hence $R R_{\tau}$ heavily depend on the threshold $\varepsilon$ which therefore must be adapted carefully to the problem at hand.

The ordinal average recurrence rate can be exactly determined:

$$
R R=\sum_{\pi} p_{\pi}^{2}
$$

where $p_{\pi}=n_{\pi} / N$ is the relative and $n_{\pi}$ the absolute frequency of the order patterns $\pi$ in the time series. To explain this formula, we note that $\mathbf{R}_{i, j}=1$ if $\pi_{i}=\pi_{j}$. For every order pattern $\pi$, the number of pairs $(i, j)$ with $\pi_{i}=\pi_{j}=\pi$ equals $n_{\pi}^{2}$. Thus the number of entries $\mathbf{R}_{i, j}=1$ in the matrix is $\sum n_{\pi}^{2}$ where the sum runs over all possible order patterns $\pi$. This fact together with (16) implies equation (17).

Let us take order patterns of length 3 as an example, and assume they all appear with equal probability. Then one sixth of the entries of the matrix $\mathbf{R}$ are 1. In Section 6, we use (17) to express coupling in the multivariate case where we cannot work with $\tau$.

For $l \geq 1$, let $P(l)$ denote the number of (maximal) diagonal line segments of length $=l$ in $\mathbf{R}$. Since they represent $l$ successive recurrences, we can introduce two measures of repeated recurrence, or strength of recurrence:

$$
D E T=\frac{\sum_{l=l_{\min }}^{N-1} l P(l)}{\sum_{i \neq j} \mathbf{R}_{i, j}}, \quad L=\frac{\sum_{l=l_{\min }}^{N-1} l P(l)}{\sum_{l=l_{\min }}^{N-1} P(l)}
$$

$D E T$ is the fraction of recurrence points on lines of length $\geq l_{\min }$, where $l_{\min }$ is a parameter $\geq 2$, and is called determinism since it increases with the predictability of the system. $L$ is the average length of a diagonal line of length $\geq l_{\min }$. Rules for choosing $l_{\min }$ can be found in [3].

If the time series is long enough, these two parameters can also be studied as functions of $\tau$. So let $P_{\tau}(l)$ denote the number of diagonal lines of exact length $l$ on the diagonal $\mathbf{R R}_{i, i+\tau}$, and 


$$
D E T_{\tau}=\frac{\sum_{l=l_{\min }}^{N-\tau} l P_{\tau}(l)}{\sum_{l=1}^{N-\tau} l P_{\tau}(l)}, \quad L_{\tau}=\frac{\sum_{l=l_{\min }}^{N-\tau} l P_{\tau}(l)}{\sum_{l=l_{\min }}^{N-\tau} P_{\tau}(l)} .
$$

\section{Cross-recurrence}

The diagonal-wise determination of the recurrence measures is useful for the study of interrelations and synchronization. For the study of interrelations we can use CRPs. Long diagonal structures in CRPs reveal a similar time evolution of the trajectories of the two processes under study. An increasing similarity between the processes causes an increase of the recurrence point density along the main diagonal $\mathbf{C R}_{i, i}(i=1 \ldots N)$. When the processes become identical, the main diagonal appears and the CRP becomes an RP. Thus, the occurrence of diagonal lines in CRPs can be used in order to benchmark the similarity between the considered processes. Using this approach it is possible to assess the similarity in the dynamics of two different systems in dependence on a certain time delay [25].

The cross-recurrence rate of a CRP

$$
R R_{\tau}=R R_{\tau}^{\boldsymbol{x}, \boldsymbol{y}}=\frac{1}{N-\tau} \sum_{i=1}^{N-\tau} \mathbf{C R}_{i, i+\tau}
$$

reveals the probability of the occurrence of similar states in both systems with a certain delay $\tau$. The average recurrence rate $R R=R R^{\boldsymbol{x}, \boldsymbol{y}}$ is determined as in (16). It depends not only on $\varepsilon$, but also indicates whether trajectories of the two systems often visit the same phase space regions.

Stochastic and strongly fluctuating processes generate only short diagonals, whereas deterministic processes often admit longer diagonals. If two deterministic processes have the same or similar time evolution, i. e. parts of the phase space trajectories visit the same phase space regions for certain times, the amount of longer diagonals increases and the amount of shorter diagonals decreases. The measures $D E T_{\tau}$ and $L_{\tau}$ of a CRP describe the similar time evolution of the systems' states.

As cross-correlation, cross-recurrence is not symmetric in $\tau$. It is possible to define indices of symmetry and asymmetry (for a small range $0 \leq \tau \ll N$ ), as

$$
Q(\tau)=\frac{R R_{\tau}+R R_{-\tau}}{2}, \quad \text { and } \quad q(\tau)=\frac{R R_{\tau}-R R_{-\tau}}{2} .
$$

By means of these indices it is possible to quantify interrelations between two systems and determine which system leads the other one (this is similar to the approach for the detection of event synchronization proposed in [28]).

Summarizing, we can state that high values of $R R_{\tau}$ indicate a high probability of occurrence of the same state in both systems, and high values of $D E T_{\tau}$ and $L_{\tau}$ indicate a long time span, in which both systems visit the same region of phase space. The consideration of an additional CRP 


$$
\mathbf{C R}_{i, j}^{-}=\Theta\left(\varepsilon-\left\|\boldsymbol{x}_{i}+\boldsymbol{y}_{j}\right\|\right)
$$

with a negative signed second trajectory $-\boldsymbol{y}_{j}$ allows distinguishing correlations and anti-correlations between the considered trajectories [25].

\section{Synchronization}

\section{Phase synchronization on metric scale}

The concept of recurrence can be used to detect indirectly phase synchronization (PS) in a wide class of chaotic systems and also systems corrupted by noise, where other methods are not so appropriate [27]. The distances between diagonal lines in an RP reflect the characteristic time scales of the system. In contrast to periodic dynamics, for a chaotic oscillator the diagonal lines are interrupted due to the divergence of nearby trajectories. Furthermore, the distances between the diagonal lines are not constant, i. e. we find a distribution of distances, reflecting the different time scales present in the chaotic system.

If two oscillators are in PS, the distances between diagonal lines in their respective RPs coincide, because their phases, and hence their time scales adapt to each other. However, the amplitudes of oscillators, which are only PS but not in general or complete synchronization, are in general uncorrelated. Therefore, their RPs are not identical. However, if the probability that the first oscillator recurs after $\tau$ time steps is high, then the probability that the second oscillator recurs after the same time interval will be also high, and vice versa. Therefore, looking at the probability $p(\tau)$ that the system recurs to the $\varepsilon$-neighborhood of a former point $\boldsymbol{x}_{i}$ of the trajectory after $\tau$ time steps and comparing $p(\tau)$ for both systems allows detecting and quantifying PS properly. As mentioned above, $p(\tau)$ can be estimated as recurrence rate, (15), $\hat{p}(\tau)=R R_{\tau}$. Studying the coincidence of the positions of the maxima of $R R_{\tau}$ for two coupled systems $\boldsymbol{x}$ and $\boldsymbol{y}$, PS can be identified. More precisely, the correlation coefficient between $R R_{\tau}^{x}$ and $R R_{\tau}^{y}$

$$
C P R=\left\langle\widetilde{R R}_{\tau}^{x} \cdot \widetilde{R R}_{\tau}^{y}\right\rangle,
$$

can be used to quantify PS. Here $\widetilde{R R}_{\tau}^{x}$ denotes $R R_{\tau}^{x}$ normalized to zero mean and standard deviation one. If both systems are in PS, the probability of recurrence will be maximal at the same time and $C P R \approx 1$. On the other hand, if the systems are not in PS, the maxima of the probability of recurrence will not occur simultaneously. Then we observe a drift and hence expect low values of $C P R$.

\section{General synchronization on metric scale}

It is also possible to detect generalized synchronization (GS) by means of RPs [27]. Let us consider the average probability of recurrence over time 
for systems $\boldsymbol{x}$ and $\boldsymbol{y}$, i. e. the recurrence rate, $R R^{\boldsymbol{x}}$ and $R R^{y}$, determined by (16). The average probability of joint recurrence over time is then given by $R R^{\boldsymbol{x}, \boldsymbol{y}}$, which is the recurrence rate of the JRP of the systems $\boldsymbol{x}$ and $\boldsymbol{y}$ [22]. If both systems are independent, the average probability of the joint recurrence will be $R R^{\boldsymbol{x}, \boldsymbol{y}}=R R^{\boldsymbol{x}} R R^{\boldsymbol{y}}$. On the other hand, if both systems are in GS, we expect approximately the same recurrences, and hence $R R^{\boldsymbol{x}, \boldsymbol{y}} \approx$ $R R^{x}=R R^{y}$. For the computation of the recurrence matrices in the case of essentially different systems that undergo GS, it is more appropriate to use a fixed amount of nearest neighbors $N_{\mathrm{n}}$ for each column in the matrix than using a fixed threshold, which corresponds to the original definition of RPs by Eckmann et al. [20]. $R R^{x}$ and $R R^{y}$ are then equal and fixed by $N_{\mathrm{n}}$, because of $R R^{x}=R R^{y}=N_{\mathrm{n}} / N$. Now we call $R R=N_{\mathrm{n}} / N$ and define the coefficient

$$
S=\frac{R R^{x, y}}{R R}
$$

as an index for GS that varies from $R R$ (independent) to 1 (GS). Furthermore, in order to be able to detect also lag synchronization (LS) [23], a time lag is included by using the time delayed JRP, Eq. (10),

$$
S(\tau)=\frac{\frac{1}{N^{2}} \sum_{i, j}^{N} \mathbf{J R}_{i, j}^{\boldsymbol{x}, \boldsymbol{y}}(\tau)}{R R} .
$$

Then, we introduce an index for GS based on the average joint probability of recurrence $J P R$ by choosing the maximum value of $S(\tau)$ and normalizing it,

$$
J P R=\max _{\tau} \frac{S(\tau)-R R}{1-R R} .
$$

The index $J P R$ ranges from 0 to 1 . The parameter $R R$ has to be fixed to compute $J P R$, but it can be shown that the $J P R$ index does not depend crucially on the choice of $R R[27]$.

\section{Phase synchronization on ordinal scale}

As mentioned before, there exists a connection between the order patterns and the phase of a signal. This connection is illustrated in Fig. 5, which suggests a representation of the oscillatory behavior of the Rössler system by order patterns. In this section we show how the order patterns of dimension $m=3$ and the common phase definitions are mathematically related.

Following [16] we introduce a new cylindrical coordinate system $(r, \phi, z)$ in terms of the time-delayed coordinates $\left(u_{i}, u_{i+\vartheta}, u_{i+2 \vartheta}\right)$. The $z$-coordinate corresponds to the main diagonal, and $r$ and $\phi$ span a plane perpendicular to the main diagonal. The radius $r$ describes the distance to the main diagonal and $\phi$ the angle. Hence, the order pattern is completely determined by $\phi$. On the other hand, the order patterns can be considered as a discretization of 
$\phi$. It has been shown in [16] that $\phi$ can be written in terms of time-delayed coordinates

$$
\tan \phi_{i}=\sqrt{3} \frac{u_{i+2 \vartheta}-u_{i}}{u_{i+2 \vartheta}-2 u_{i+\vartheta}+u_{i}} \approx 2 \sqrt{3} \frac{\dot{u}_{i+1}}{\ddot{u}_{i+1}} .
$$

Several concepts have been introduced to define a phase for chaotic oscillators $[29,30]$. Nevertheless these approaches are in general restricted to narrowband signals. For this reason, alternative methods based on the curvature of a phase-space-trajectory have been proposed [31, 32], where the phase is defined as $\phi=\arctan \dot{x} / \dot{y}$. In a similar sense, a phase can be defined as $\phi^{\prime}=\arctan \dot{x} / \ddot{x}$, which coincides with relation (26), up to a constant factor.

To derive a measure for phase synchronization we analyze the coincidence of phases of two oscillators by means of the recurrence rate $R R_{\tau}$ of order patterns. This yields a distribution of phase differences as a function of the time-lag $\tau$. Following the idea of [33], we introduce a coupling index by means of the Shannon entropy

$$
\rho_{\pi}=1-\frac{-\sum_{\tau=\tau_{\min }}^{\tau_{\max }} r r_{\tau} \log r r_{\tau}}{\log \left(\tau_{\max }-\tau_{\min }\right)},
$$

where $r r_{\tau}$ is the normalized distribution $r r_{\tau}=R R_{\tau} / \sum_{\tau} R R_{\tau}$. This index ranges from 0 to 1 , where 0 indicates that both systems are independent from each other. The actual maximum depends on $\left[\tau_{\min }, \tau_{\max }\right]$, if there are several maxima with distance of a mean recurrence time. Due to a close relationship between the order patterns and the phase, we expect that $\rho_{\pi}$ is sensitive to phase synchronization.

This connection to phase indicates a main difference between recurrence plots on metric and ordinal scale. In case of a phase-coherent but chaotic oscillator such as the Rössler system the trajectory returns irregularly to itself. A metric recurrence plot as Fig. 4 has only short diagonals. But due to a high coherence of the phase the recurrence time is narrow-banded, and the recurrence rate shows sharp equidistant peaks (cf. Fig. 9). Considering recurrence on ordinal scale, only the phase is taken into account. Hence we observe long lines in the recurrence plot (Fig. 5), while the distances of peaks in the recurrence rate coincide with that of the metric case.

\section{Prototypical examples}

\section{Finding nonlinear interrelations using cross recurrence}

This example shows the ability of CRPs to find nonlinear interrelations between two processes, which cannot be detected by means of linear tests [25]. We consider linear correlated noise (auto-regressive process of order 1 , see for example [34]) which is nonlinearly coupled with the $x$-component of the 

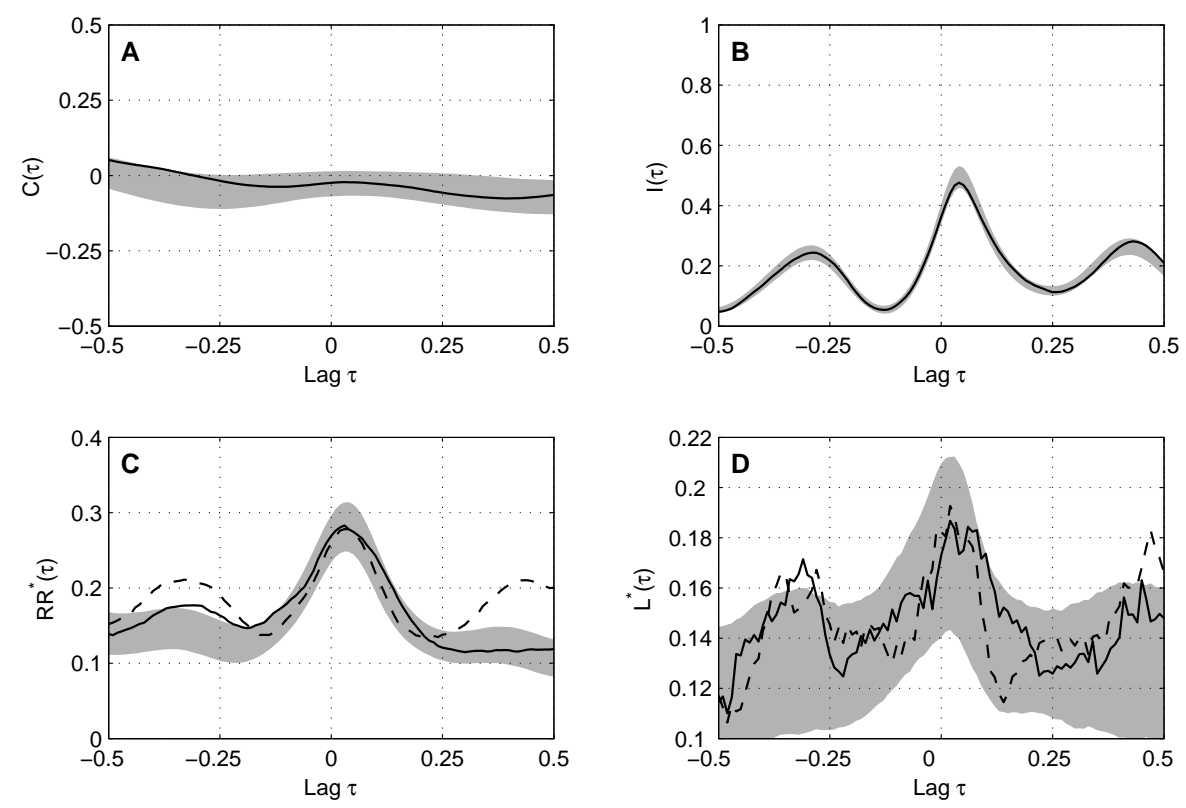

Fig. 8. (A) Cross correlation $C(\tau)$, (B) mutual information $I(\tau),(\mathrm{C}) \tau$-recurrence rate $R R_{\tau}$ ofr the model given in (28). (D) $\tau$-average line length $L_{\tau}$ for the forced auto-regressive process and the forcing function; the curves represent the measures for one realization as functions of the delay $\tau$ for a coupling $\kappa=0.2$. In (C) and (D) the solid lines show positive relation; the dashed lines show negative relation. The grey bands mark the $2 \sigma$ margin of the distributions of the measures gained from 500 realizations. The lag $\tau$ and the average line length $L_{\tau}$ have units of time [25].

Lorenz system (for standard parameters $\sigma=10, r=28, b=8 / 3$ and a time resolution of $\Delta t=0.01[35,36])$ :

$$
y_{i}=0.86 y_{i-1}+0.500 \xi_{i}+\kappa x_{i}^{2},
$$

where $\xi$ is Gaussian white noise and $x_{i}\left(x(t) \rightarrow x_{i}, t=i \Delta t\right)$ is normalized with respect to the standard deviation. The data length is 8,000 points and the coupling $\kappa$ is realized without any lag.

As expected, due to the nonlinear relationship, the cross correlation analysis between $x$ and $y$ does not reveal any significant linear correlation between these data series (Fig. 8A). However, the mutual information as a well-established measure to detect nonlinear dependencies [37] shows a strong dependence between $x$ and $y$ at a delay of 0.05 (Fig. 8B). The CRP based $\tau$-recurrence rate $R R_{\tau}$ and $\tau$-average diagonal length $L_{\tau}$ exhibit maxima at a lag of about 0.05 for $R R^{+} / L^{+}$and $R R_{\tau}^{-} / L_{\tau}^{-}$and additionally at 0.45 and -0.32 for $R R_{\tau}^{-} / L_{\tau}^{-}$(Figs. $8 \mathrm{C}, \mathrm{D}$ ). The maxima around 0.05 for the + and - measures are a strong indication of a nonlinear relationship between the data. The delay of approximately 0.05 stems from the auto-correlation of $y$ 
and approximately corresponds to its correlation time $\Delta t / \ln 0.86=0.066$. The maxima at 0.45 and -0.32 correspond to half of the mean period of the Lorenz system. Since the result is rather independent of the sign of the second data, the found interrelation is of the kind of an even function. 500 realizations of the AR model have been used in order to estimate the distributions of the measures. The $2 \sigma$ margins of these distributions can be used to assess the significance of the results.

Due to the rapid fluctuations of $y$, the number of long diagonal lines in the CRP decreases. Therefore, measures based on these diagonal structures, especially $D E T_{\tau}$, do not perform well on such heavily fluctuating data. However, we can infer that the measures $R R_{\tau}$, as well as $L_{\tau}$ (though less significant for rapidly fluctuating data), are suitable for finding a nonlinear relationship between the considered data series $x$ and $y$, where the linear analysis is not able to detect such a relation. In contrast to mutual information, this technique is applicable to rather short and non-stationary data.
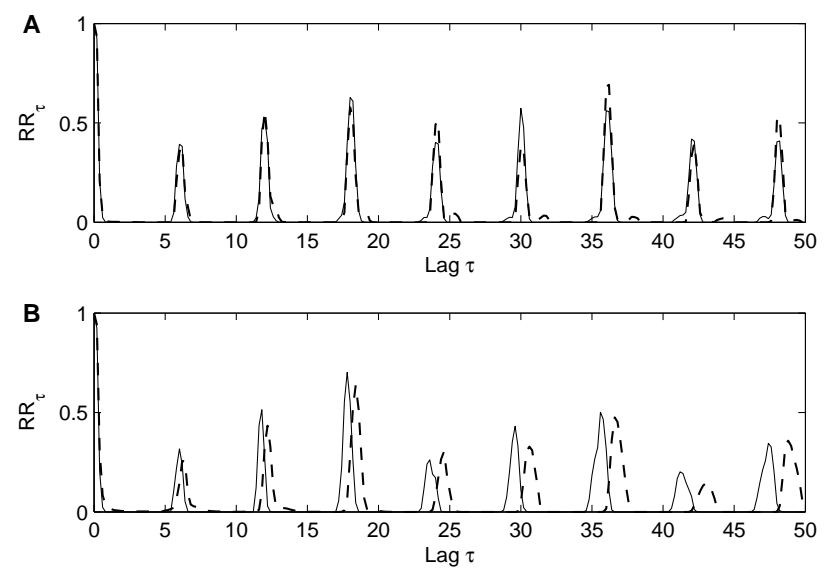

Fig. 9. Recurrence probability $R R_{\tau}$ for two mutually coupled Rössler systems, Eqs. (29) and (30), for $a=0.16, b=0.1, c=8.5$, in (A) phase synchronized and (B) non-phase synchronized regime. Solid line: oscillator $\boldsymbol{x}$, dashed line: oscillator $\boldsymbol{y}$.

\section{Synchronization in Rössler oscillators: metric scale}

In order to exemplify this method, we consider two mutually coupled Rössler systems

$$
\begin{aligned}
& \dot{x}_{1}=-(1+\nu) x_{2}-x_{3}, \\
& \dot{x}_{2}=(1+\nu) x_{1}+a x_{2}+\mu\left(y_{2}-x_{2}\right), \\
& \dot{x}_{3}=b+x_{3}\left(x_{1}-c\right),
\end{aligned}
$$




$$
\begin{aligned}
& \dot{y}_{1}=-(1-\nu) y_{2}-y_{3}, \\
& \dot{y}_{2}=(1-\nu) y_{1}+a y_{2}+\mu\left(x_{2}-y_{2}\right), \\
& \dot{y}_{3}=b+y_{3}\left(y_{1}-c\right) .
\end{aligned}
$$

in the phase coherent regime $(a=0.16, b=0.1, c=8.5)$, similar to the example of Fig. 4. According to [31], for $\nu=0.02$ and $\mu=0.05$ both systems are in PS. We observe that the local maxima of $R R_{\tau}^{x}$ and $R R_{\tau}^{y}$ occur at $\tau=n T$, where $T$ is the mean period of both Rössler systems and $n$ is an integer (Fig. 9A).
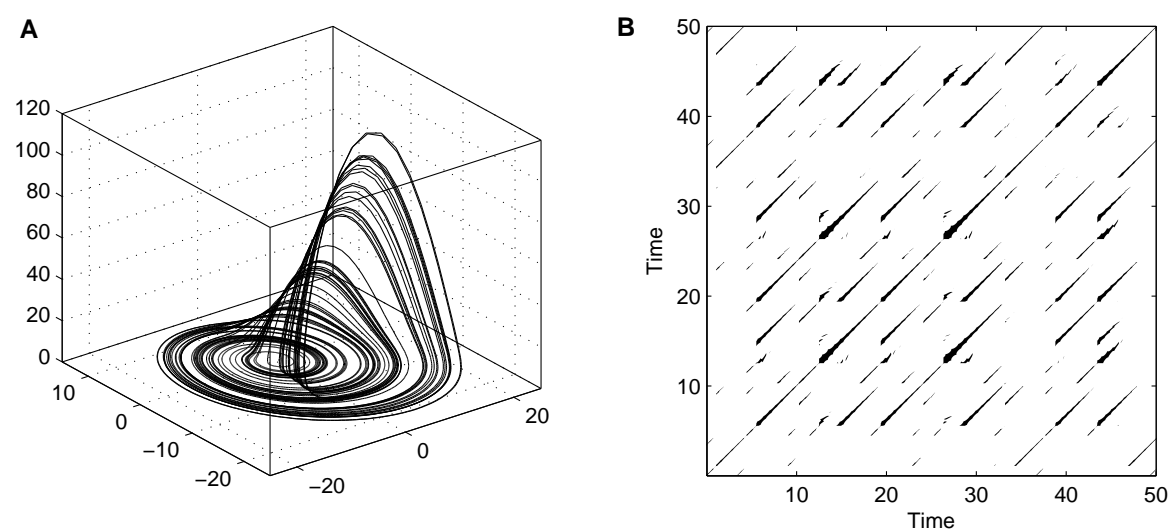

Fig. 10. (A) Trajectory and (B) Recurrence plot for a Rössler system in the funnel regime, Eqs. (14) for $a=0.2925, b=0.1, c=8.5$. Compare with Fig. 4.

Note that the heights of the local maxima are in general different for both systems if they are only in PS (and not in stronger kinds of synchronization, such as generalized or complete synchronization [30]). But the positions of the local maxima of $R R_{\tau}$ coincide, and the correlation coefficient is $C P R=0.998$. For $\mu=0.02$ the systems are not in PS and the positions of the maxima of $R R_{\tau}$ do not coincide anymore (Fig. 9B), clearly indicating that the frequencies are not locked. In this case, the correlation coefficient is $C P R=0.115$.

It is important to emphasize that this method is highly efficient even for non-phase coherent oscillators, such as two mutually coupled Rössler systems in the rather complicated funnel regime, Eqs. (29) and (30), for $a=0.2925$, $b=0.1, c=8.5, \nu=0.02$ (Fig. 10). We analyze again two different coupling strengths: $\mu=0.2$ and $\mu=0.05$. The peaks in $R R_{\tau}$ (Fig. 11) are not as wellpronounced and regular as in the coherent regime, reflecting the different time scales that play a relevant role and the broad band power spectrum of these systems. However, for $\mu=0.2$ the positions of the local maxima coincide for both oscillators (Fig. 11A), indicating PS, whereas for $\mu=0.05$ the positions 

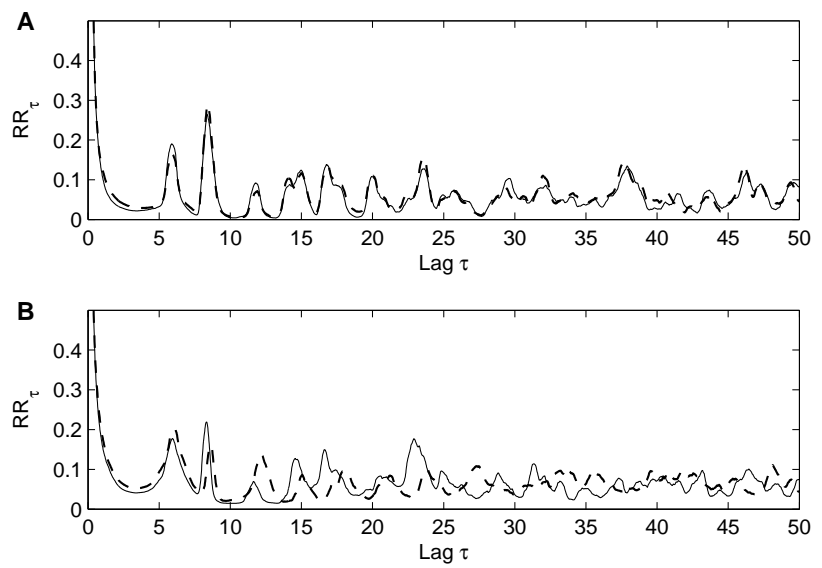

Fig. 11. Recurrence probability $R R_{\tau}$ for two mutually coupled Rössler systems in funnel regime, Eqs. (29) and (30), for $a=0.2925, b=0.1, c=8.5$. (A) $\mu=0.2$ (PS) and (B) $\mu=0.05$ (non-PS). Solid line: oscillator $\boldsymbol{x}$, dashed line: oscillator $\boldsymbol{y}$.

of the local maxima do not coincide anymore (Fig. 11B), indicating non-PS. These results are in accordance with [31].

In the PS case of this latter example, the correlation coefficient is $C P R=$ 0.988 , and in the non-PS case, $C P R=0.145$. Note that the positions of the first peaks in $R R_{\tau}$ coincide (Fig. 11B), although the oscillators are not in PS. This is due to the small frequency mismatch $(2 \nu=0.04)$. However, by means of the index $C P R$ we can distinguish rather well between both regimes.

Furthermore, the index $C P R$ is able to detect PS even in time series which are strongly corrupted by noise [27]. Additionally, $C P R$ indicates clearly the onset of PS. In [27], the results obtained for $C P R$ in dependence on the coupling strength were compared with the Lyapunov exponents, as they theoretically indicate the onset of PS (in the phase-coherent case). The results obtained with $C P R$ coincide with the ones obtained by means of the Lyapunov exponents.

The results obtained with $C P R$ are very robust with respect to the choice of the threshold $\varepsilon$. Simulations show that the outcomes are almost independent of the choice of $\varepsilon$ corresponding to a percentage of black points in the RP between $1 \%$ and $90 \%$, even for non-coherent oscillators. The patterns obtained in the RP, of course, depend on the choice of $\varepsilon$. But choosing $\varepsilon$ for both interacting oscillators in such a way that the percentage of black points in both RPs is the same, the relationship between their respective recurrence structures does not change for a broad range of values of $\varepsilon$. 


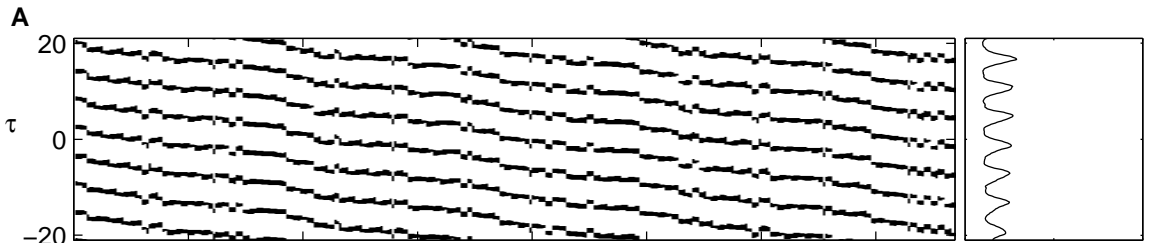

B

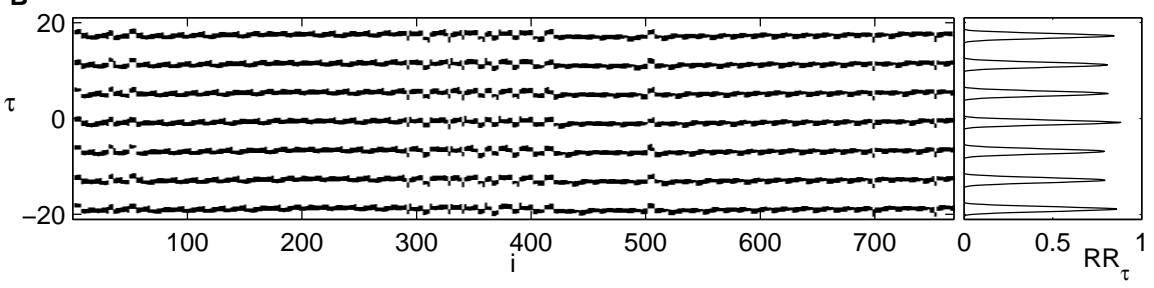

Fig. 12. Cross recurrence plot on ordinal scale and corresponding recurrence rate $R R_{\tau}$ for two mutually coupled Rössler systems in (A) non-phase synchronized and (B) phase synchronized regime. Embedding parameters are $m=3$ and $\vartheta=2$.

\section{Synchronization in Rössler oscillators: ordinal scale}

The locking of phases in case of synchronized oscillators is also reflected by order patterns, which then become synchronized, too. The order patterns represent the phase, which allows an instantaneous study of phase interaction during the onset of phase synchronization of oscillators. A direct comparison of states has the main advantage to study synchronization behavior instantaneously. In order to study a longer range in time and to focus on a small range of the time-lag we choose a slightly different representation of the recurrence plot. In the following we consider the recurrence plot as a function of time $i$ and time-lag $\tau=i-j$, where diagonal lines become horizontal lines.

Fig. 12 shows cross recurrence plots on ordinal scale of two mutually coupled Rössler systems. The parameters are the same as before. In the non-phase synchronized regime (Fig. 12A, $\mu=0.02$ ) both oscillators diverge due to detuning and consequently we observe drifting lines. The corresponding recurrence rate $R R_{\tau}$ shows no significant values. In case of phase synchronization both oscillators pass the regions of order patterns simultaneously, which is reflected in long horizontal lines (Fig. $12 \mathrm{~B}, \mu=0.05$ ). The recurrence rate shows distinct peaks with a distance of the mean recurrence time. With metric CRPs we do also observe the transition to PS, but the lines are interrupted because amplitudes are not equal, as they would be in complete synchronization.

In the non-phase-coherent funnel regime (Fig. 10) the distribution of recurrence times is broad, what is already reflected in the recurrence rate on metric scale (Fig. 11). In case of no phase synchronization $(\mu=0.05)$ we observe only very short horizontal lines in the cross recurrence plot on ordinal scale (Fig. 13A), and the recurrence rate shows no significant values. In case 

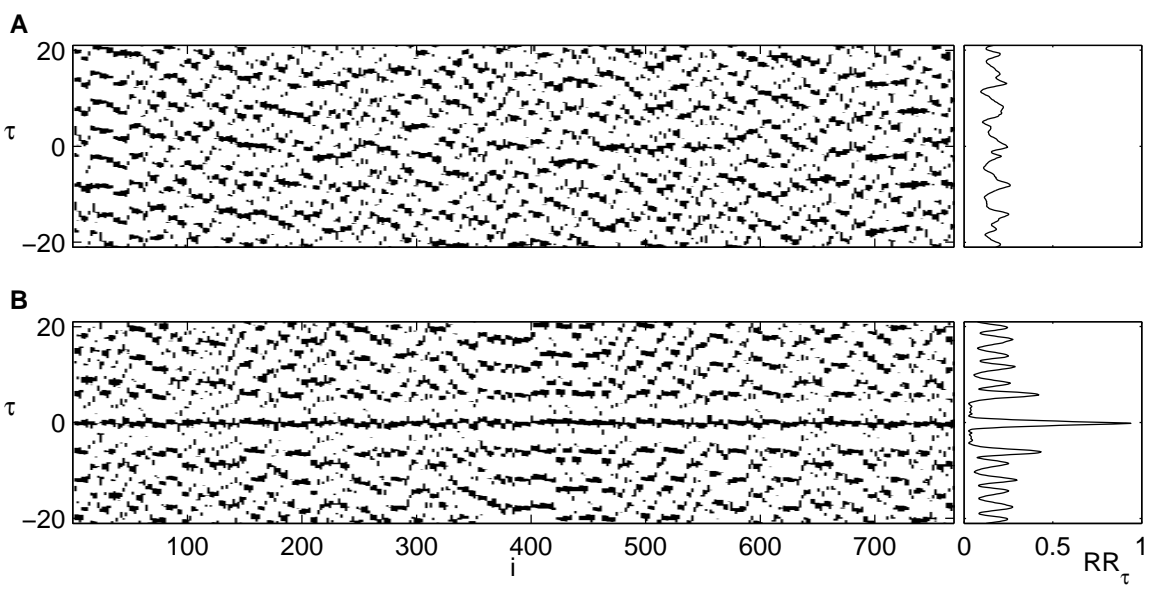

Fig. 13. Cross recurrence plot on ordinal scale and corresponding recurrence rate $R R_{\tau}$ for two mutually coupled Rössler systems in funnel regime in (A) non-phase synchronized and (B) phase synchronized regime. Embedding parameters are $m=3$ and $\vartheta=2$.

of phase synchronization (Fig. $13 \mathrm{~B}, \mu=0.2$ ) the plot clearly shows a long line at $\tau \approx 0$. But in contrast to the phase-coherent regime, there are no other distinct lines and the recurrence rate exhibits only a single dominant peak.

\section{Application to EEG data}

\section{Synchronization analysis during an epileptic seizure}

The following application to EEG data illustrates the advantages of an analysis on ordinal scale in contrast to the metric scale. Scalp EEG data are susceptible to many artifacts which cause offset and amplitude fluctuations. We study the phenomenon of synchronization of neuronal groups during an epileptic seizure, where the specific type of phase synchronization has already been discussed to detect seizure activity [38].

We consider EEG signals from a 14-year old child with epileptic disorder, which were provided by H. Lauffer from the Department of Pediatric Medicine of the University of Greifswald. The 19-channel EEG data (international 1020 system) were sampled with $256 \mathrm{~Hz}$ and band-pass filtered $(0.3-70 \mathrm{~Hz})$. On two representative channels the seizure onset is shown in Fig. 14A, indicated by a gray bar.

The data during the seizure are clearly dominated by oscillations in the alpha range $(\approx 8-13 \mathrm{~Hz})$ which are locked, indicating synchronization. This yields to a high coupling index $\rho_{\pi}$ of the order patterns (Fig 14B). Before the seizure there are no dominant oscillations, and the coupling index $\rho_{\pi}$ 


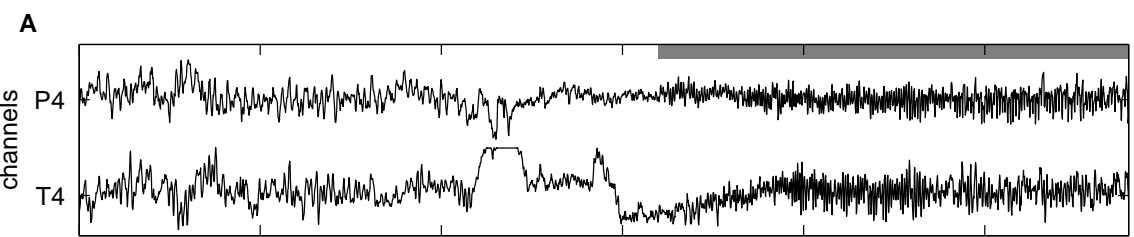

B

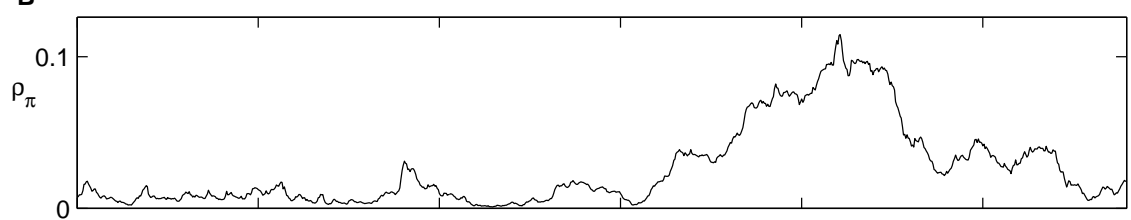

C

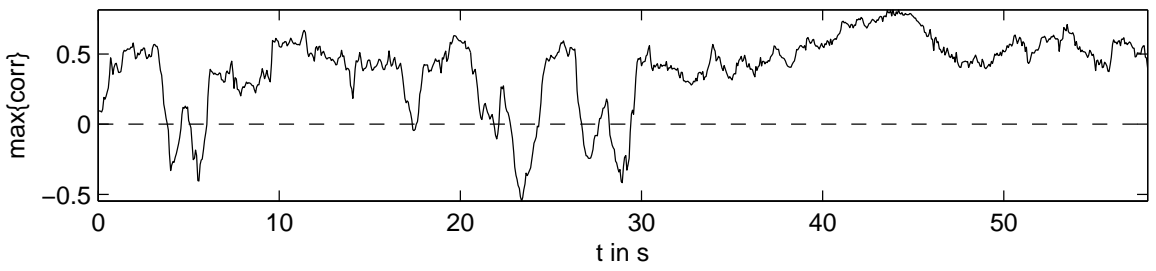

Fig. 14. (A) Coupling of two EEG channels during onset of epileptic seizure (gray bar). (B) Coupling index $\rho_{\pi}$ derived from ordinal recurrence plot with embedding parameters $m=3$ and $\vartheta=27 \mathrm{~ms}$. (C) Maximum value of cross-correlation function. Both coupling indices are determined in a sliding window of length $2 \mathrm{~s}$. Considered time-lag between both channels $-0.2 s \ldots 0.2 s$.

is clearly smaller. Although the EEG data are corrupted by artifacts, the coupling index gives reliable results to reveal the seizure period. The crosscorrelation function, however, is strongly disturbed (Fig. 14C), and special pre-processing of the data would be inevitable for its use.

\section{Multivariate coupling in event-related potentials}

Our second example will show that quantization of coupling makes sense also for data which do not oscillate. Event-related potentials are multivariate time series which show the reaction of the human brain on a certain stimulus [39]. They are non-stationary, but since the experiment is repeated many times, we have a whole sample of such time series. The traditional way is to take the average time series and look for its maxima and minima which are then compared with peaks of typical reaction curves, called N100 or P300 since they appear 100 or $300 \mathrm{~ms}$ after the stimulus and are negative or positive.

It is not clear whether these most obvious peaks represent the most interesting brain activity, but there is little fine structure in an average curve of hundreds of time series. Today's challenge is the analysis of single trials, 
and the huge problem is that beside the reaction under study, there is a lot of other brain activity, measurement errors etc.
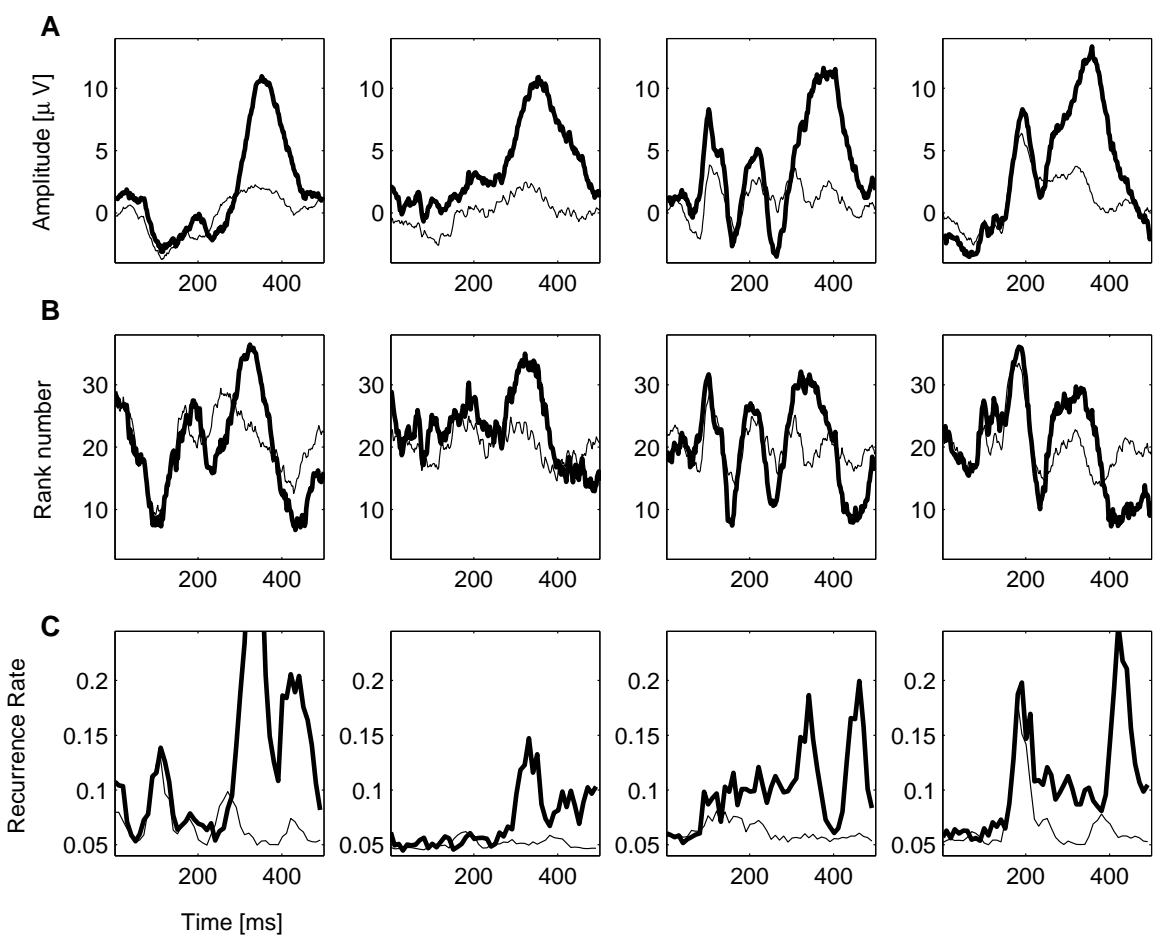

Fig. 15. Reaction of four persons (columns) in a visual oddball experiment. (A) Mean amplitude of 9 parietal channels and all ordinary (thin) resp. oddball (thick) trials clearly shows P300. (B) Corresponding means of rank numbers (40 values backwards) show the effect less clearly. (C) The recurrence rate of rank numbers for all nine channels measures the multivariate coupling of the channels. P300 comes out very clearly, but a bit different from (A). Note also N100 for the first person and P200 for the fourth.

The question we address here is whether certain reactions can be characterized not only by the size of amplitudes, but also by the coupling of different channels, with no regard to the size of values. This is recent research of C. Bandt and D. Samaga with A. Hamm and M. Weymar from the Department of Psychology in Greifswald who performed a simple oddball experiment just for single-trial analysis. Eight male students were presented, in intervals of about two seconds, 150 equal shapes on a screen. 23 were red, and 127 yellow, and the red (oddball) patterns had to be counted. Raw EEG data with sample frequency $500 \mathrm{~Hz}$ were taken from 128 channels (Electrical Geodesics, Inc.). Preprocessing involved subtraction of the average of all channels, se- 
lection of 54 important channels in the parietal and occipital region, and a decent projection method to remove the $50 \mathrm{~Hz}$ mains hum.

Theory predicts that the rare oddball patterns, on which the attention is directed, are characterized by a clear P300 peak in parietal channels - in the average curve. This was true, and is shown for the first four subjects and the average of 9 parietal channels in Fig. 15A. Note that there are big differences between individuals, which casts doubt on the common practice to take "grand averages" over many persons. For instance, the first person has a distinctive N100, and the last one has a clear P200.
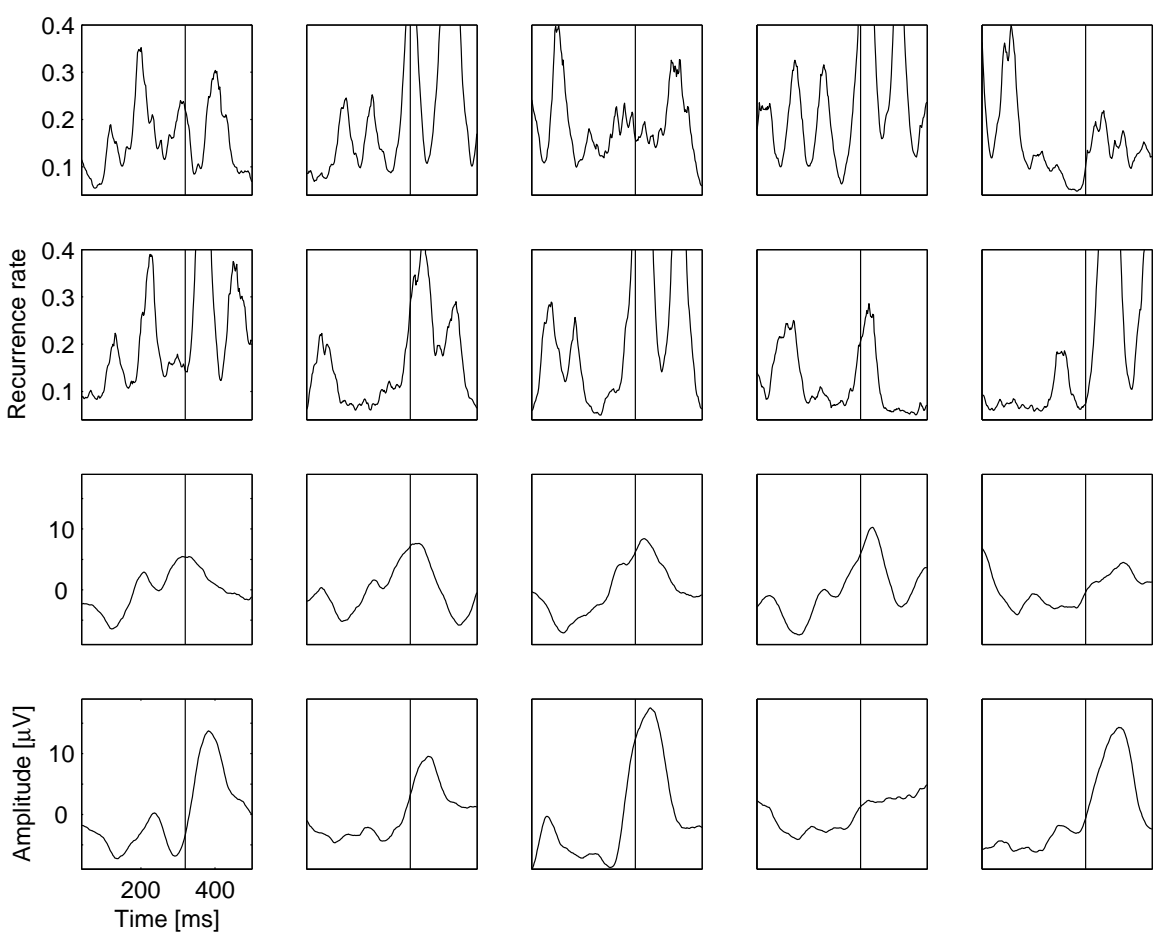

Fig. 16. Analysis of P300 for the first 10 oddball trials of the first person. The two upper rows show recurrence rates of order patterns, over 9 channels and time windows of $40 \mathrm{~ms}$, measuring the coupling of the channels. The two lower rows show average amplitude, also taken over 9 channels and $40 \mathrm{~ms}$ windows. Time from 40 to $500 \mathrm{~ms}$, vertical lines at $300 \mathrm{~ms}$. As in Fig. 15, P300 usually has a single peak in amplitude and a double peak in coupling.

The question was whether order patterns in the time series will also detect the oddball effect. We took rank numbers with respect to the previous 40 values (80 ms), $r_{i}=\#\left\{k \mid 1 \leq k \leq 40, x_{i-k}<x_{i}\right\}$ [14]. The resulting average curves were worse than amplitudes in characterizing oddball trials (Fig. 15B). 
This is because those rank numbers represent the local structure of the curve: even a huge P300 value in one trial, which influences the average in Fig. 15A, can only have rank number 40, which means it is just larger than the last 40 values. Other features, however, as N100 for the first person, seem to come out more properly with rank numbers than with amplitudes.
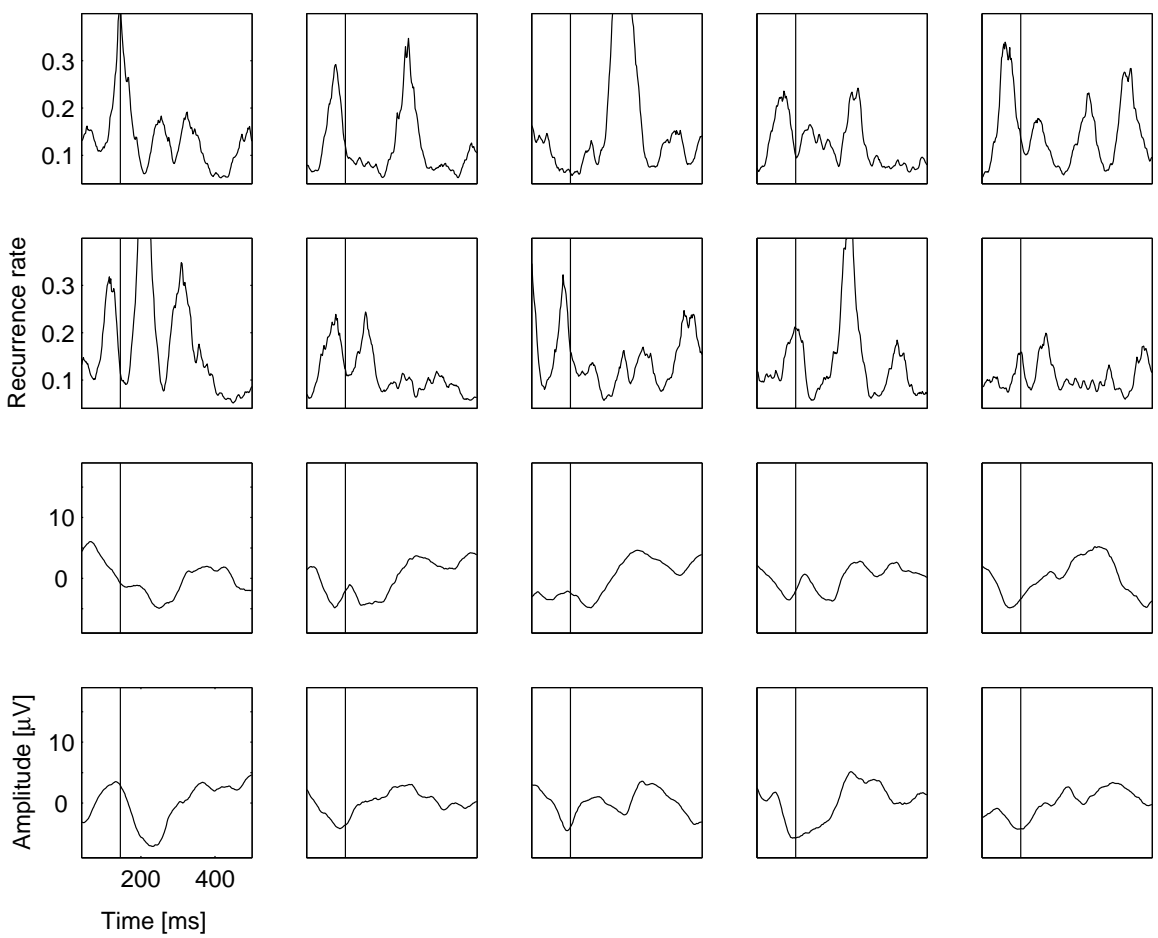

Fig. 17. Single-trial analysis of N100 for the first 10 ordinary trials of the first person. The two upper rows show recurrence rates of order patterns over 9 channels and time windows of $40 \mathrm{~ms}$, measuring the coupling of the channels. The two lower rows show average amplitude for the corresponding trials. Time from 40 to $500 \mathrm{~ms}$, vertical lines at $130 \mathrm{~ms}$. To detect N100, coupling performed better than amplitude.

Now we took order patterns of length $m=4$, with lag $\vartheta=22 \mathrm{~ms}$, for these 9 channels in the parietal region, and all red (resp. yellow) trials, and took the recurrence rate of the resulting distribution of order patterns, as defined in (17). To have a better statistics for the 23 red trials, we also took disjoint windows of 5 successive time points $(10 \mathrm{~ms}$ ). (When we estimated $4 !=24$ probabilities $p_{\pi}$ from $9 \cdot 23$ order patterns for each time point, the curve became rather noisy, so we took always 5 time points together.) $\mathrm{RR}$ is high when many patterns coincide, so it measures the coupling of the channels. The result in Fig. 15C shows that this coupling really distinguishes the oddball 
trials, at least in the overall statistics. The same was found for permutation entropy [17], and also for the rank numbers instead of order patterns.

Now let us go to single trials, taking only the first person. Fig. 16 shows the first 10 oddball trials. The recurrence rates were taken over the 9 parietal channels and over sliding windows of 20 points. For one time point in a single trial, we have only 9 order patterns, one for each channel. With the sliding window, we had $9 \cdot 20=180$ order patterns to estimate $4 !=24$ probabilities $p_{\pi}$ which resulted in surprisingly smooth curves. For a fair comparison, the amplitudes in the lower part of Fig. 16 were also averaged over the 9 channels and sliding windows of width 20 . The P300 can be seen in most cases, with a single peak in amplitude and a twin peak in the coupling. This connection deserves further attention.

As was indicated in Fig. 15, the trials also show N100 - small amplitude and coupling around $100 \mathrm{~ms}$. This will be our main concern when we now study the first 10 ordinary trials. There is a peak in coupling around $100 \mathrm{~ms}$ in 9 of 10 trials of Fig. 17. In the corresponding pictures of amplitude, it is less obvious that there is a minimum. At this point recurrence rates work better. Comparing Figs. 16 and 17, we see that the reaction at $100 \mathrm{~ms}$ comes later in oddball trials.

Ordinal trials also show weak P300 peaks, which is possible from a physiological point of view. In fact we had to accumulate the information from many channels to obtain rules for every individual which correctly classify oddball and ordinary trials in $90 \%$ of all cases. Other peaks in Fig. 17 which irregularly appear may have to do with further brain activity. On the whole, this example indicates that coupling concepts can be very useful for single-trial analysis.

\section{Acknowledgement}

This study was made possible by support from the DFG priority programme SPP 1114.

\section{References}

1. K. Petersen. Ergodic Theory. Cambridge University Press, 1983.

2. J. P. Zbilut and C. L. Webber, Jr. Embeddings and delays as derived from quantification of recurrence plots. Physics Letters A, 171(3-4):199-203, December 1992.

3. N. Marwan, M. C. Romano, M. Thiel, and J. Kurths. Recurrence Plots for the Analysis of Complex Systems. Physics Reports, 438(5-6):237-329, 2007.

4. R. Durbin, S. Eddy, A. Krogh, and G. Mitchison. Biological Sequence Analysis. Cambridge University Press, 1998.

5. J.-P. Eckmann, S. O. Kamphorst, and D. Ruelle. Recurrence plots of dynamical systems. Europhysics Letters, 4:973-977, Nov 1987. 
6. F. Takens. Lecture Notes in Mathematics, volume 898, chapter Detecting strange attractors in turbulence, pages 366-381. Springer, Berlin, 1981.

7. T. Sauer, J. A. Yorke, and M. Casdagli. Embedology. Journal of Statistical Physics, 65(3 - 4):579-616, 1991.

8. S. S. Stevens. On the theory of scales of measurement. Science, 103:677-680, June 1946.

9. M. Hallin and J. Jurečkova. Optimal tests for autoregressive models based on autoregression rank scores. The Annals of Statistics, 27(4):1385-1414, 1999.

10. S. Frisch, P. beim Graben, and M. Schlesewsky. Parallelizing grammatical functions: P600 and p345 reflect different cost of reanalysis. International Journal of Bifurcation and Chaos, 14(2):531-549, 2004.

11. A. Goettlein and H. Pruscha. Advances in GLIM and statistical modeling, volume 78, chapter Ordinal time series models with application to forest damage data, pages 113-118. Springer, 1992.

12. M. G. Kendall and J. D. Gibbons. Rank Correlation Methods. Oxford University Press, New York, 5th edition, 1990.

13. T. S. Ferguson, C. Genest, and M. Hallin. Kendall's tau for serial dependence. The Canadian Journal of Statistics, 28(3):587-604, Sep 2000.

14. C. Bandt. Ordinal time series analysis. Ecological Modelling, 182(3-4):229-238, 2005.

15. C. Bandt and F. Shiha. Order patterns in time series. Journal of Time Series Analysis, 2007.

16. A. Groth. Visualization of coupling in time series by order recurrence plots. Physical Review E, 72(4):046220, 2005.

17. C. Bandt and B. Pompe. Permutation entropy: A natural complexity measure for time series. Physical Review Letters, 88:174102, 2002.

18. C. Bandt, G. Keller, and B. Pompe. Entropy of interval maps via permutations. Nonlinearity, 15:1595-1602, 2002.

19. J. M. Amig, M. B. Kennel, and L. Kocarev. The permutation entropy rate equals the metric entropy rate for ergodic information sources and ergodic dynamical systems. Physica D, 210(1-2):77-95, 2005.

20. J.-P. Eckmann, S. O. Kamphorst, and D. Ruelle. Recurrence Plots of Dynamical Systems. Europhysics Letters, 5:973-977, 1987.

21. M. Thiel, M. C. Romano, P. L. Read, and J. Kurths. Estimation of dynamical invariants without embedding by recurrence plots. Chaos, 14(2):234-243, 2004.

22. M. C. Romano, M. Thiel, J. Kurths, and W. von Bloh. Multivariate Recurrence Plots. Physics Letters A, 330(3-4):214-223, 2004.

23. G. Rosenblum, A. S. Pikovsky, and J. Kurths. From Phase to Lag Synchronization in Coupled Chaotic Oscillators. Physical Review Letters, 78(22):4193-4196, 1997.

24. O. V. Sosnovtseva, A. G. Balanov, T. E. Vadivasova, V. V. Astakhov, and E. Mosekilde. Loss of lag synchronization in coupld chaotic systems. Physical Review E, 60(6), 1999.

25. N. Marwan and J. Kurths. Nonlinear analysis of bivariate data with cross recurrence plots. Physics Letters A, 302(5-6):299-307, 2002.

26. O. E. Rössler. An equation for continuous chaos. Physics Letters A, 57(5):397398, 1976.

27. M. C. Romano, M. Thiel, J. Kurths, I. Z. Kiss, and J. Hudson. Detection of synchronization for non-phase-coherent and non-stationary data. Europhysics Letters, 71(3):466-472, 2005. 
28. R. Quian Quiroga, T. Kreuz, and P. Grassberger. Event synchronization: A simple and fast method to measure synchronicity and time delay patterns. Physical Review E, 66(4):041904, 2002.

29. M. Rosenblum, A. Pikovsky, and K. Kurths. Phase synchronization of chaotic oscillators. Physical Review Letters, 76(11):1804-1807, 1996.

30. A. Pikovsky, M. Rosenblum, and J. Kurths. Synchronization - A universal concept in nonlinear sciences. Cambridge University Press, 2003.

31. G. Osipov, B. Hu, C. Zhou, M. Ivanchenko, and J. Kurths. Three types of transition to phase synchronization in coupled chaotic oscillators. Physical Review Letters, 91(2):024101, 2003.

32. I. Z. Kiss, Q. Lv, and J. L. Hudson. Synchronization of non-phase coherent chaotic electrochemical oscillations. Physical Review E, 71:035201, 2005.

33. P. Tass, M. Rosenblum, J.Weule, J. Kurths, A. Pikovsky, J. Volkmann, A. Schnitzler, and H.-J. Freund. Detection of n:m phase locking from noisy data: Application to magnetoencephalography. Physical Review Letters, 81(15):32913294, 1998.

34. R. H. Shumway and D.S. Stoffer. Time Series Analysis and Its Applications. Springer, New York, 2006.

35. E. N. Lorenz. Deterministic Nonperiodic Flow. Journal of the Atmospheric Sciences, 20:120-141, 1963.

36. J. H. Argyris, G. Faust, and M. Haase. An Exploration of Chaos. North Holland, Amsterdam, 1994.

37. H. Kantz and T. Schreiber. Nonlinear Time Series Analysis. Cambridge University Press, second edition, reprint edition, 2005.

38. F. Mormann, K. Lehnertz, P. David, and C. E. Elger. Mean phase coherence as a measure for phase synchronization and its application to the eeg of epilepsy patients. Physica D Nonlinear Phenomena, 144:358-369, October 2000.

39. T. C. Handy (ed.). Event-Related Potentials. MIT Press, Cambridge, Mass., 2005. 